BASIC RESEARCH

\title{
Optical coherence tomographic elastography technique for measuring deformation and strain of atherosclerotic tissues
}

\author{
J Rogowska, N A Patel, J G Fujimoto, M E Brezinski
}

Heart 2004;90:556-562. doi: 10.1136/hrt.2003.016956

See end of article for authors' affiliations

....................

Correspondence to: Dr M E Brezinski, Orthopedics Department, Brigham and Women's Hospital/Harvard Medical School, 75 Francis St., Boston, Massachusetts 02115 , USA; mebrezin@ mit.edu

Accepted

1 September 2003

\begin{abstract}
Objectives: To evaluate optical coherence tomographic elastography as a method for assessing the elastic properties of atherosclerotic plaque and the parameters that influence interpretation.

Methods: Phantoms and aorta were examined in vitro to quantify speckle modulation and measure the displacement and strain maps. A correlation method was used as a speckle tracking technique for measuring axial and lateral displacement vectors and calculation of strain maps. The influence of correlation kernel size on accuracy of the method was evaluated.

Results: In terms of a percentage error between calculated and measured displacements, the best results for phantoms were obtained with a $41 \times 41$ kernel (1.88\% error). For both phantom and aorta images, it was found that, with the increasing size of cross correlation kernel, the axial and lateral displacement maps are less noisy and the displacement vectors are more clearly defined. However, the large kernels tend to average out the differences in displacements of small particles in phantoms and decrease the ability of speckle tracking to make microstructural assessments. Therefore, it is important to select kernel size carefully, based on the image features.

Conclusions: Optical tomographic elastography can be used to assess the microstructural properties of atherosclerotic tissue at micrometre scale resolution, but preselected analysis criteria must be understood in a critical interpretation of the results.
\end{abstract}

M ost myocardial infarctions and other acute coronary syndromes are caused by unstable plaques in the coronary arteries that are relatively small, contain lipids, and are thin walled. When these plaques rupture, they release thrombogenic material into the blood, which results in clot formation and potential vessel occlusion. These vulnerable plaques are a diagnostic dilemma in clinical medicine for several reasons. Firstly, these plaques are beyond the detection limit of any available imaging modalities. Secondly, most plaques that rupture, often referred to as a vulnerable plaque, do not appear to lead to acute unstable coronary syndromes. In other words, most are asymptomatic. This means that we need a better understanding of which thin walled or vulnerable plaques lead to acute coronary syndromes. For instance, it has been noted that reduced collagen content in plaque walls may make the plaque structurally weak and more likely to lead to a coronary syndrome.

Recently, identification of vulnerable plaque in vitro and in vivo has been shown to be feasible with optical coherence tomography (OCT)..$^{1-8}$ OCT is analogous to ultrasound, measuring the intensity of backreflected infrared light rather than sound. However, more information on the characteristics of the plaque other than structural imaging, such as the biomechanical properties of the plaque, may be useful in identifying unstable coronary syndromes. One technique that may be of use for the evaluation of unstable plaque mechanical properties is OCT elastography. We examined the value of OCT elastography for the assessment of atherosclerotic tissue.

Elastography is a process of estimating biomechanical (elastic) properties of tissues through imaging techniques. By applying a known pressure on the tissue under study, tissue stiffness can be detected and tissue strain determined. Since pathological tissues typically have different biomechanical properties from normal tissues, elastography can help monitor pathological states, such as abnormal weakening of vessel walls. Therefore, the elastic properties of tissues, together with other properties, such as structure identified from backreflection intensity, can be helpful in making a diagnosis.

Ultrasound elastography, which has been under development for over 10 years, exploits local changes in the elastic modulus of tissue as a contrast mechanism for improving the detection of lesions. ${ }^{9-13}$ In general, with ultrasound elastography, a stress is applied to the tissue and the resulting strain pattern is estimated. The strain induced in hard tissue is smaller than that in softer tissues. From the resultant deformation pattern, the local strain is estimated assuming that the applied stress is uniform or has a known distribution.

Another elastography technique, magnetic resonance elastography, is also used for measuring tissue strain. ${ }^{14-18}$ The magnetic resonance elastography technique is noninvasive and sensitive to motion but is relatively expensive, since the high magnetic field environment requires specially designed equipment.

Recently, OCT elastography has been demonstrated using speckle tracking techniques. ${ }^{19-20}$ A primary strength of OCT based elastography is the potential to evaluate the mechanics of intact tissue with a higher spatial resolution (10-100 times) than ultrasound, which enables a more precise characterisation of tissues and, therefore, a more accurate assessment of microscale variations of elastic properties. Schmitt ${ }^{19}$ measured the displacements as small as a few micrometres in heterogeneous gelatin phantoms containing scattering particles (latex spheres) in addition to living skin. His results, however, also indicate that better models are needed to transform the displacement images into quantitative maps of subtle regional variations of elastic modulus.

We evaluated OCT elastography as a method for assessing the elastic properties of atherosclerotic plaque, standardised with phantoms. More importantly, the study examined with 
phantoms and in vitro aorta how pre-chosen variables, such as kernel size, affect the assessment of tissue elastic properties when analysing speckle modulation.

\section{METHODS}

\section{Experimental elastography system}

The theory behind OCT has been previously described. OCT is analogous to ultrasound, measuring the intensity of backreflected infrared light. ${ }^{2}$ Ultrashort light pulses or low coherent light is generated at the sample. The time for the light to be reflected back or echo delay time is used to measure distances. The intensity of backreflection is plotted as a function of depth. The beam is then scanned across the sample to produce two and three dimensional data sets. Figure 1 illustrates the experimental elastography system, where the samples (phantom or aorta) were placed inside a holder (Petri dish) and covered with a cover slip.

\section{Phantom and aorta images}

Phantoms were made by mixing $\mathrm{lg}$ of agarose and $8 \mathrm{~g}$ gelatin, and dissolving it in $50 \mathrm{ml}$ of boiling water. The mixture was stirred for several minutes until all clumps were dissolved and then $0.25 \mathrm{~g}$ of activated charcoal was added. The mixture was poured into the Petri dish and refrigerated for several hours. The phantoms were cut into $25 \mathrm{~mm}$ squares, covered with a cover slip, and scanned with OCT. The elastic properties of the phantoms were confirmed by applying weight of $100 \mathrm{~g}$ and measuring changes in tissue width with callipers and OCT imaging (corrected for refractive index).

The aorta samples were obtained postmortem and stored at $0^{\circ} \mathrm{C}$ with $0.1 \%$ sodium azide. The samples were scanned with OCT before and after compression with the same procedure as phantom scanning. OCT imaging was performed on a translation stage at $1300 \mathrm{~nm}$ with an axial resolution of $18 \mu \mathrm{m}$. The spot size was $30 \mu \mathrm{m}$.

\section{Image processing techniques for calculation of displacement maps}

The pairs of precompression and postcompression images were processed with the cross correlation technique. ${ }^{19} 20$ The total displacement at a given pixel was computed by two dimensional cross correlation of precompression and postcompression media within a defined window (kernel). For a given pixel $(\mathrm{x}, \mathrm{y})$, the cross correlation coefficient (corr) between $\left(\mathrm{m}_{1} \times \mathrm{m}_{2}\right)$ pixels in area of pixel $(\mathrm{x}, \mathrm{y})$ in image $\mathrm{P}$ and a potential target region in image $\mathrm{R}$, which

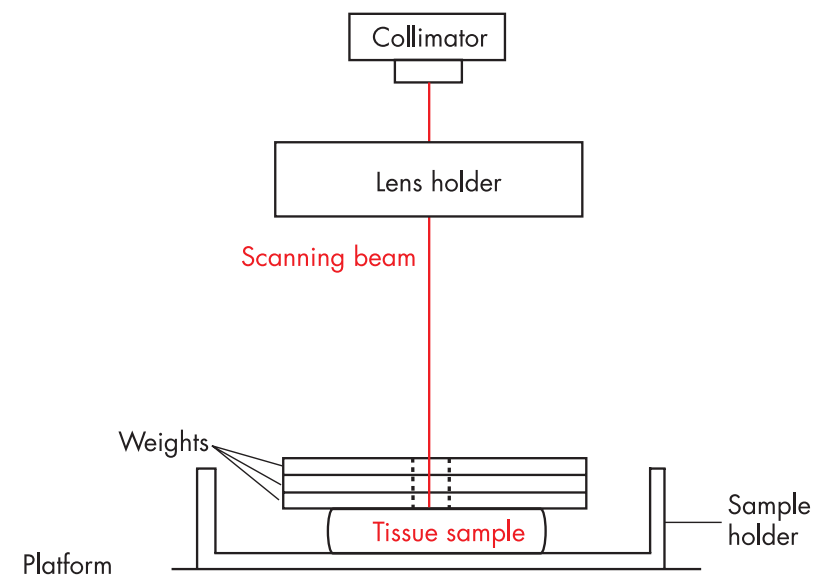

Figure 1 Diagram of the experimental optical coherence tomographic elastography system. was moved by $\mathrm{n}$ pixels axially and $\mathrm{k}$ pixels laterally, is defined by

$$
\operatorname{corr}_{x y}(n, k)=\frac{\sum_{i=1}^{m_{1}} \sum_{j=1}^{m_{2}}\left(P_{i, j}-\mu_{P}\right)\left(R_{i+n, j+k}-\mu_{R}\right)}{\sqrt{\sum_{i=1}^{m_{1}} \sum_{j=1}^{m_{2}}\left(P_{i, j}-\mu_{P}\right)^{2}\left(R_{i+n, j+k}-\mu_{R}\right)^{2}}}
$$

where $\mu_{\mathrm{P}}$ and $\mu_{\mathrm{R}}$ are the mean values in $\left(\mathrm{m}_{1} \times \mathrm{m}_{2}\right)$ areas on images $\mathrm{P}$ and $\mathrm{R}$, respectively. The cross correlation coefficient has a maximum value of 1 for identical regions and zero value for the uncorrelated regions. Over the search region, an array of correlation coefficients is calculated. This array is the cross correlation function and its peak value identifies target destination. This procedure is repeated until the processing window moves over the entire image (or region of the image of interest).

For a given pixel $(x, y)$, the axial displacement $d_{a}(x, y)$ and lateral displacement $d_{l}(x, y)$ are defined, respectively, as values $\mathrm{A}$ and $\mathrm{L}$, for which the cross correlation function obtains maximum:

$$
\begin{aligned}
& \mathrm{d}_{\mathrm{a}}(\mathrm{x}, \mathrm{y})=\mathrm{A} \text { and } \mathrm{d}_{\mathrm{l}}(\mathrm{x}, \mathrm{y})=\mathrm{L} \text { if } \operatorname{corr}_{\mathrm{xy}}(\mathrm{A}, \mathrm{L}) \\
& =\max \left\{\operatorname{corr}_{\mathrm{xy}}(\mathrm{n}, \mathrm{k}) \text { for all } \mathrm{n} \text { and } \mathrm{m}\right\}
\end{aligned}
$$

For each pixel $(x, y)$ we can plot the displacement values $d_{a}$ and $\mathrm{d}_{1}$ and thereby obtain axial and lateral displacement maps. The axial and lateral displacement values can be combined to form vectors $\left(\mathrm{d}_{\mathrm{a}}, \mathrm{d}_{1}\right)$, which can be graphically represented as displacement vector maps.

The size of the processing kernel, $\left(m_{1} \times m_{2}\right)$, is an important parameter in this method. We have calculated the axial and lateral displacement maps for our phantom and aorta images by using the following kernel sizes: $21 \times 21$, $31 \times 31,41 \times 41,51 \times 51$, and $61 \times 61$ pixels. The displacement vectors were calculated by axial and lateral displacement arrays and were later averaged with a $5 \times 5$ spatial mean filter. For clarity, the arrows were drawn every eight pixels.

The mean and standard deviation were calculated on all axial displacement images. The calculated displacement values were compared with the measured displacement by calculating the percentage errors.

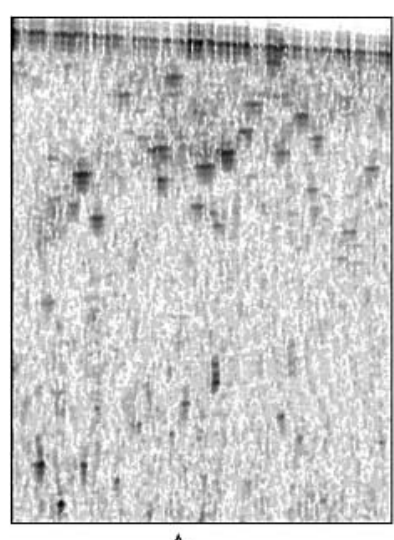

A

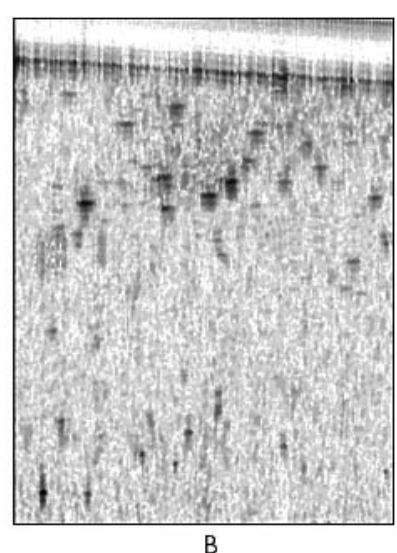

B
Figure 2 (A) Original and (B) displaced phantom images. 
A

B
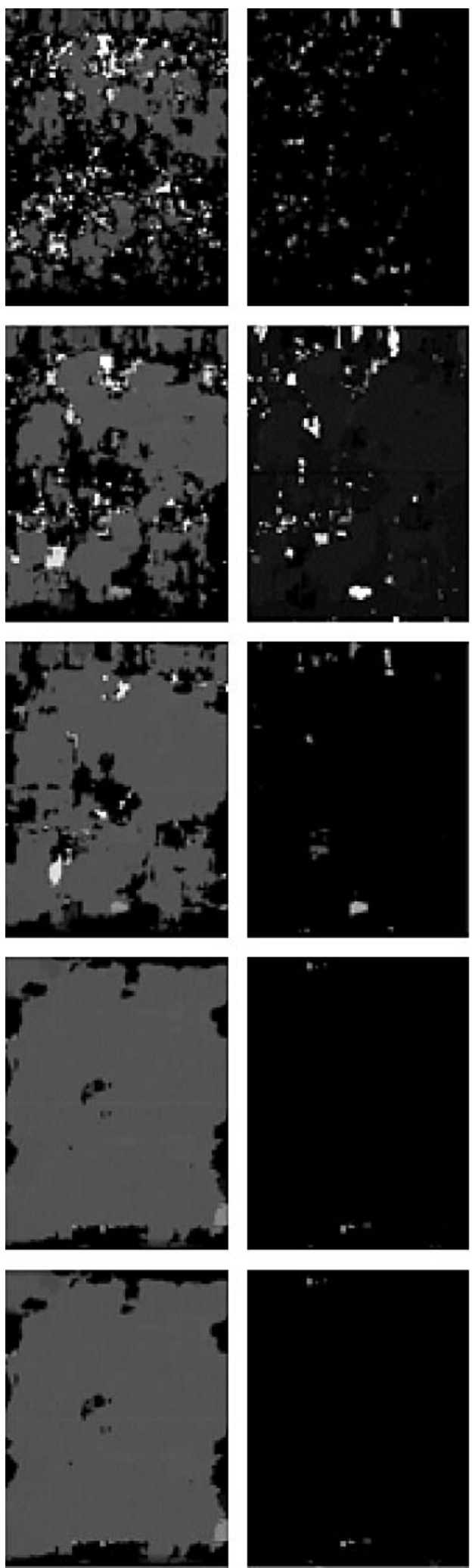

Axial

displacement
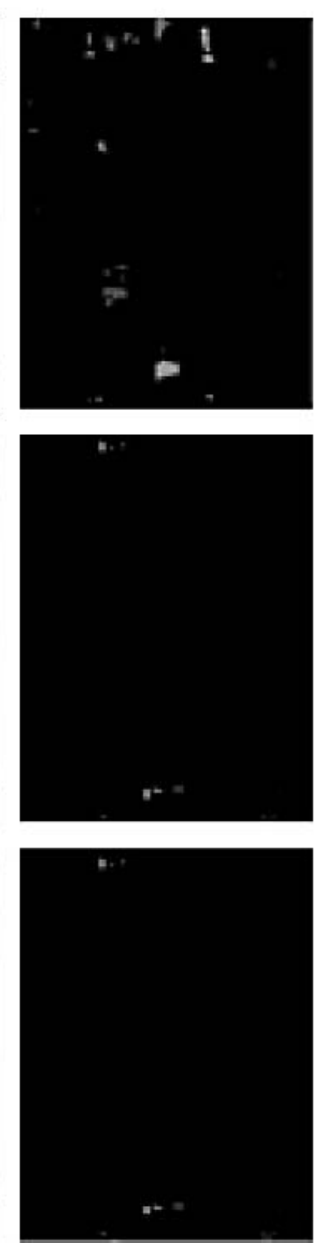

Lateral

displacement
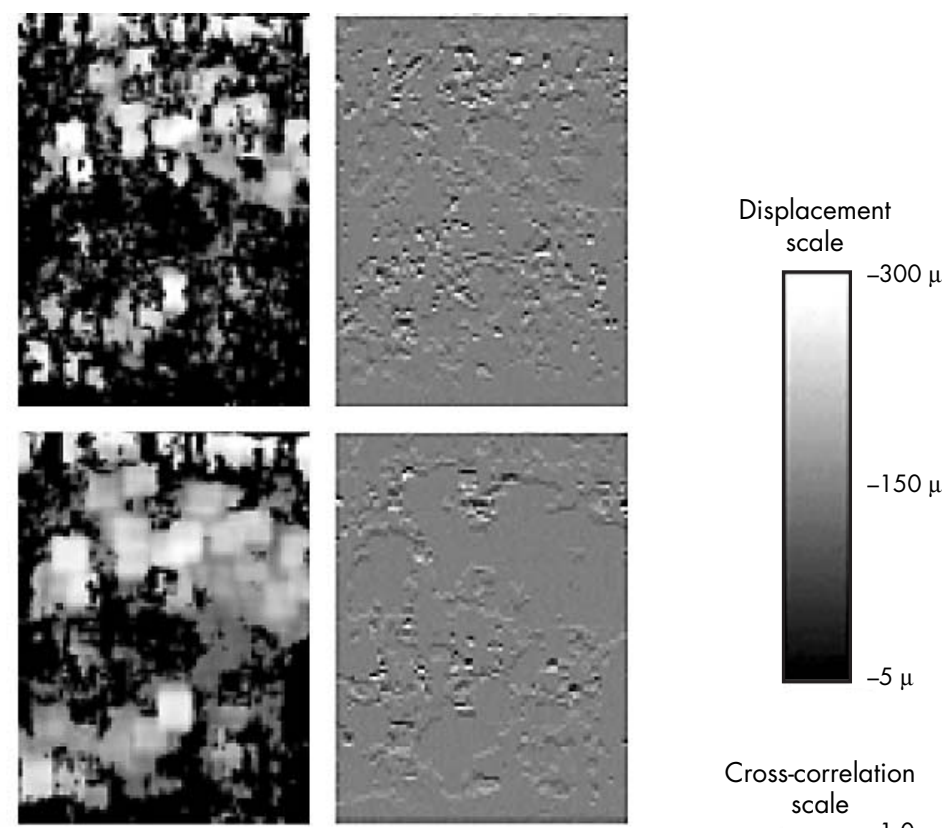

Cross-correlation scale
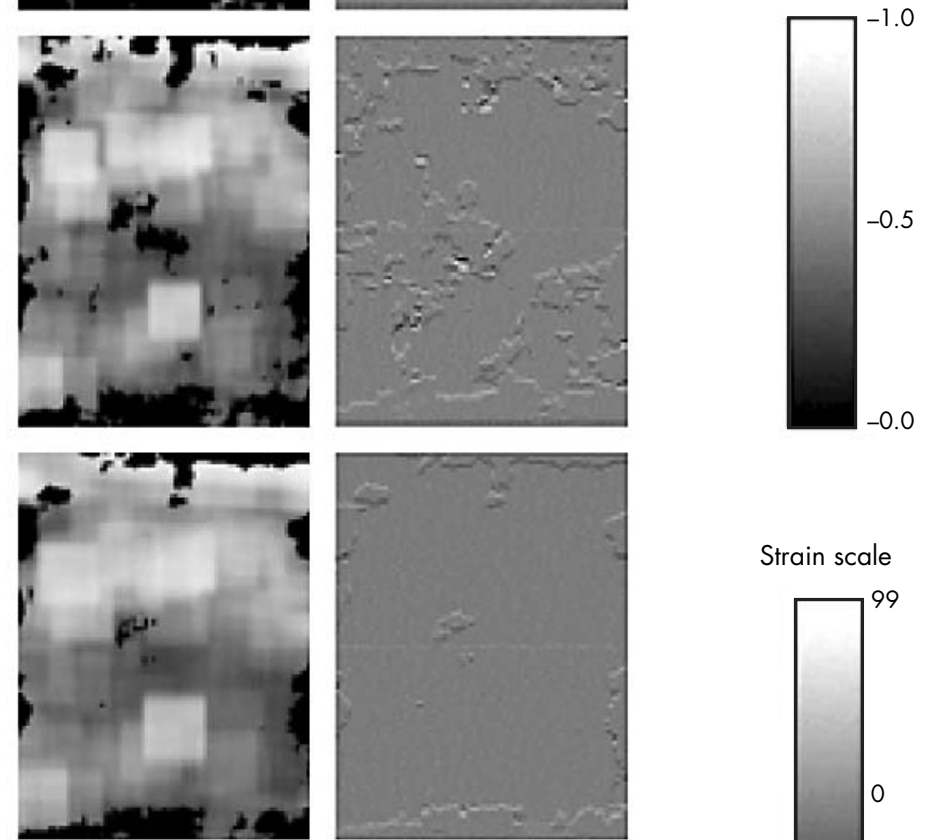

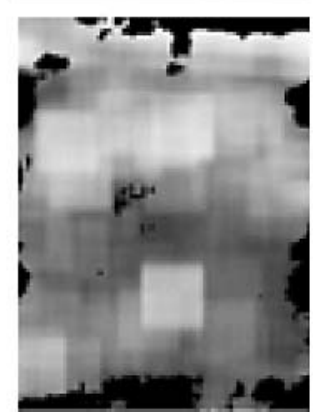

Maximum cross-correlation
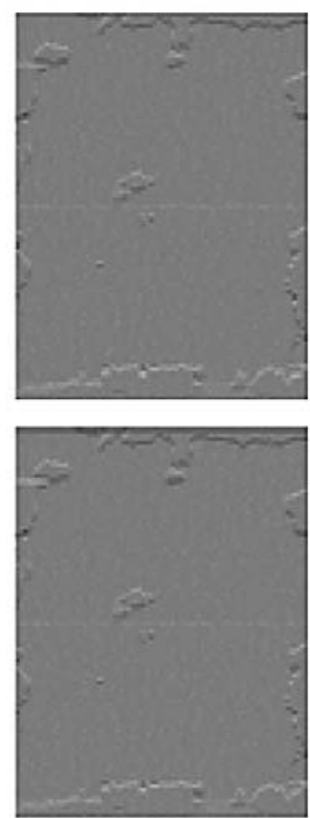

Axial strain maps

Figure 3 Axial and lateral displacements, maximum cross correlation, and strain maps calculated for phantom images on fig 2 by cross correlation with kernels of (A) $21 \times 21$, (B) $31 \times 31$, (C) $41 \times 41$, (D) $51 \times 51$, and (E) $61 \times 61$ pixels. 


\section{Calculation of strain maps}

If tissues are assumed to be uniform, isotropic, and incompressible and if the stress is applied along one axis only, the Young's modulus (or elastic modulus) E is described by:

$$
\mathrm{E}=\frac{\sigma}{\varepsilon}
$$

where $\sigma$ is the axial (or normal) stress (defined as the force perpendicular to the cross sectional area, divided by the cross sectional area; units are $\mathrm{lb} / \mathrm{in}^{2}$ or $\mathrm{N} / \mathrm{m}^{2}$ ):

$$
\sigma=\frac{\mathrm{F}}{\mathrm{A}} \quad\left(\text { units are } \mathrm{lb} / \mathrm{in}^{2} \text { or } \mathrm{N} / \mathrm{m}^{2}\right)
$$

and $\varepsilon$ is the axial strain (defined as the fractional change in length; strain has no units):

$$
\varepsilon=\frac{\Delta \mathrm{L}}{\mathrm{L}} \quad(\text { strain has no units })
$$

Our goal is to calculate the local values of elastic modulus in the phantoms. If we assume that the phantoms are uniform, isotropic, and incompressible and the stress is applied uniformly in the axial direction, then the local stress is equal to the applied stress (constant throughout the sample) and only the local strain is needed for calculation of Young's modulus.

Local strain $\hat{\varepsilon}$ is determined from an estimate of tissue axial displacement:

$$
\hat{\varepsilon}=\frac{\mathrm{d}_{2}-\mathrm{d}_{1}}{\Delta \mathrm{z}}
$$

where $d_{1}$ is the displacement estimate at a distance $\mathrm{z}$ from the top of the phantom and $\mathrm{d}_{2}$ is the displacement estimate at a distance $z+\Delta z$ from the top of the sample (the displacement values are calculated by the cross correlation technique).

The local strain can be shown as an image or map. A map of local strain is therefore an inverse map of elastic modulus; this is the way an elastogram is usually presented.
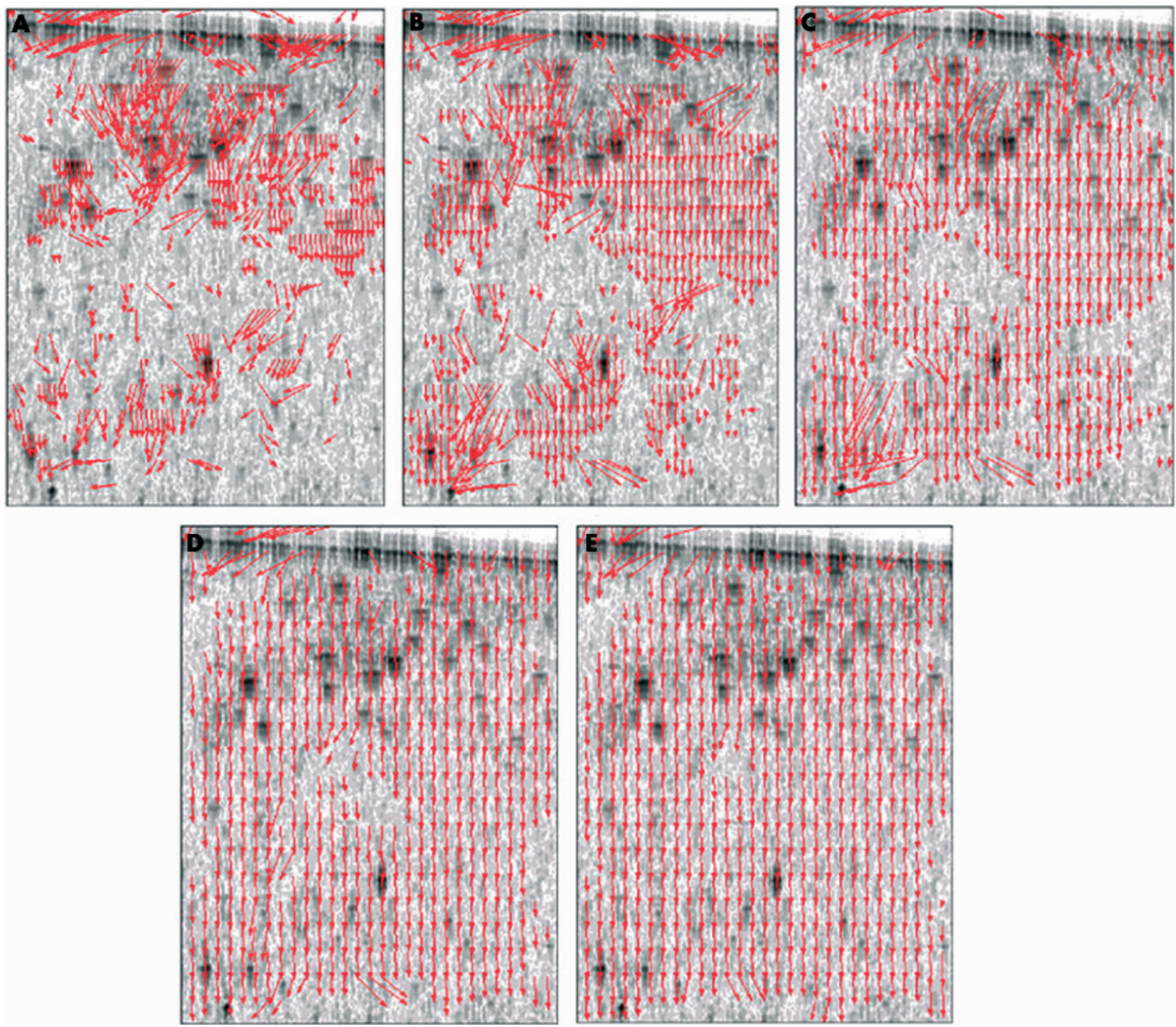

Figure 4 Displacement vectors obtained for phantom images by cross correlation with kernels (A) $21 \times 21$, (B) $31 \times 31$, (C) $41 \times 41$, (D) $51 \times 51$, and (E) $61 \times 61$ pixels. 


\begin{tabular}{|c|c|c|c|}
\hline $\begin{array}{l}\text { Kernel size } \\
\text { (pixels) }\end{array}$ & $\begin{array}{l}\text { Calculated mean axial } \\
\text { displacement }(\mu \mathrm{m})\end{array}$ & $\begin{array}{l}\text { Measured mean axial } \\
\text { displacement }(\mu \mathrm{m})\end{array}$ & $\begin{array}{l}\text { Percentage } \\
\text { error }\end{array}$ \\
\hline $21 \times 21$ & 63.53 & 94.5 & $32.77 \%$ \\
\hline $31 \times 31$ & 82.91 & 94.5 & $12.26 \%$ \\
\hline $41 \times 41$ & 96.28 & 94.5 & $1.88 \%$ \\
\hline $51 \times 51$ & 106.01 & 94.5 & $12.17 \%$ \\
\hline $61 \times 61$ & 108.96 & 94.5 & $15.30 \%$ \\
\hline
\end{tabular}

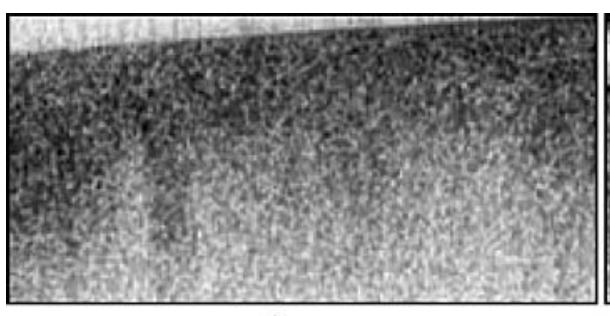

A

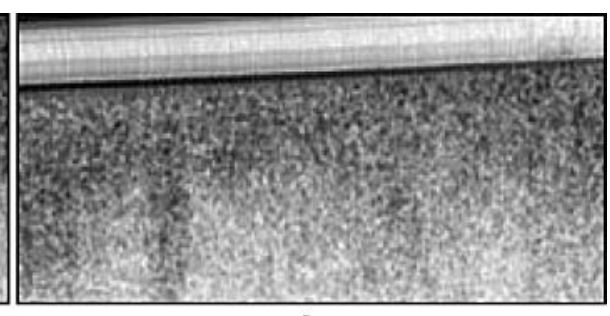

B

Figure 5 (A) Original and (B)

displaced aorta images.

All image processing techniques were implemented in Matlab (MathWorks, Natick, Massachusetts, USA).

\section{RESULTS}

The phantom images were processed by cross correlation (equation 1 ) with several kernel sizes varying from $21 \times 21$ to $61 \times 61$ pixels. Larger kernel sizes were not able to track the small charcoal particles and were not used in this study. Figure 2 presents original and displaced phantom images. Figure 3 shows the axial and lateral displacements, as well as maximum cross correlation and strain maps calculated by cross correlation with kernels of $21 \times 21,31 \times 31,41 \times 41$, $51 \times 51$, and $61 \times 61$ pixels. Figure 4 shows the corresponding displacement vectors obtained by cross correlation with kernels of $21 \times 21,31 \times 31,41 \times 41,51 \times 51$, and $61 \times 61$ pixels. Table 1 shows mean displacement values calculated for different kernel sizes and the percentage errors between calculated and measured displacements.

Fig 5 shows the original and displaced aorta images. Figures 6, 7, and 8 depict the results from the correlation
A

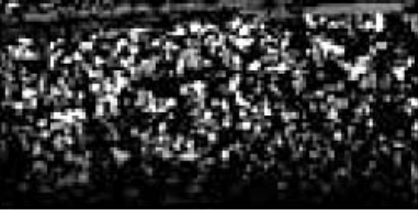

B
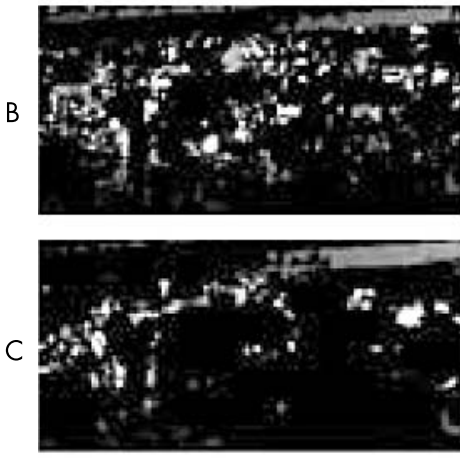

D

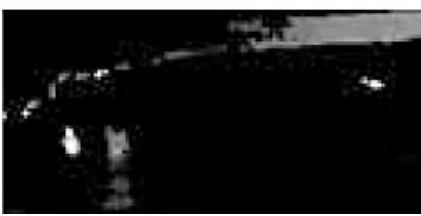

Axial displacement
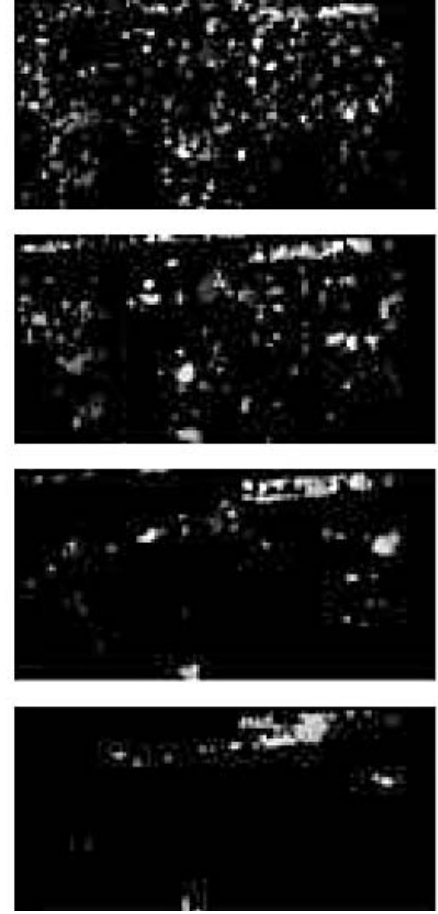

Lateral displacement
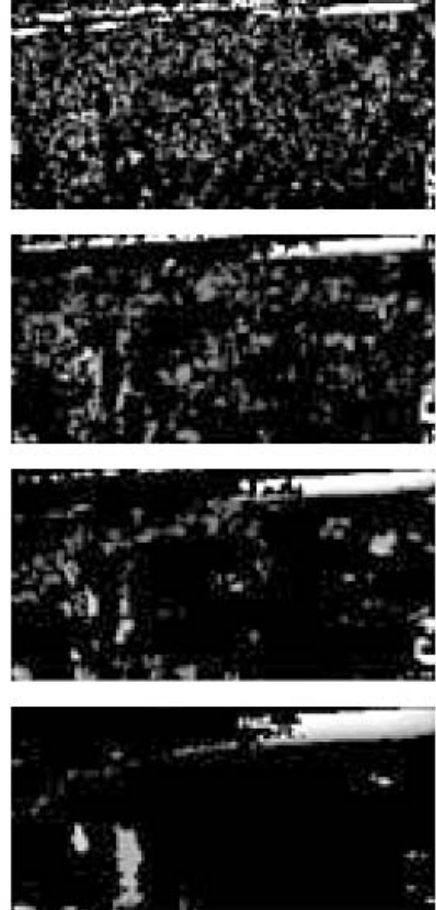

Maximum cross-correlation
Displacement scale

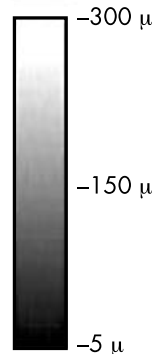

Cross-correlation scale

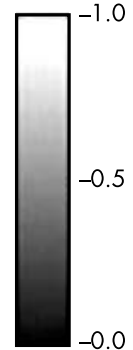

$-0.0$

Figure 6 Axial, lateral, and maximum cross correlation maps calculated for aorta images in fig 5 with the following kernels: (A) $21 \times 21$, (B) $31 \times 31$, (C) $41 \times 41$, and (D) $61 \times 61$ pixels. 
technique applied to the aorta images. Fig 6 presents the axial and lateral displacement maps and maximum cross correlation maps. Figure 7 shows the strain images. Figure 8 shows the corresponding displacement vectors obtained by cross correlation with kernels of $21 \times 21,31 \times 31,41 \times 41$, and $61 \times 61$ pixels.

\section{DISCUSSION}

Phantoms and in vitro aorta were examined to assess speckle modulation and measure the displacements and strain maps. By using phantoms, we investigated the influence of kernel size on the accuracy of the displacement measurements. In terms of a percentage error between calculated and measured displacements, the best results for phantoms were obtained with a $41 \times 41$ kernel $(1.88 \%$ error $)$. For both phantom and aorta images we found that, with the increasing size of cross correlation kernel, the axial and lateral displacement maps are less noisy and the displacement vectors are more clearly defined. However, the large kernels tend to average out the differences in displacements of small particles in phantoms and decrease the ability of speckle tracking to make microstructural assessments. Therefore, it is important to select kernel size carefully, based on the image features.

The calculation of displacement maps can also be improved. Since cross correlation is a very time consuming process, it can be modified by either a "coarse to fine" or "feature selection" technique. In the first approach, an image is divided into large blocks (perhaps overlapping) and the correlation is calculated between them (this takes just a few calculations). Only blocks with maximum cross correlation are further analysed (by cross correlation). In the feature selection technique, some important features or areas of

A

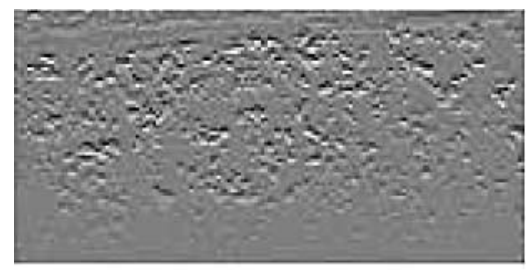

B

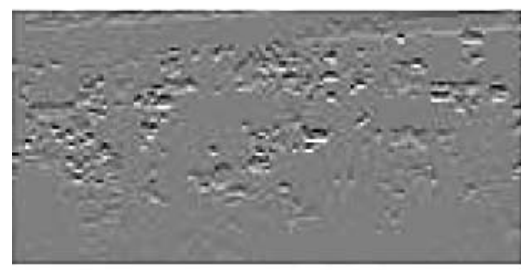

C

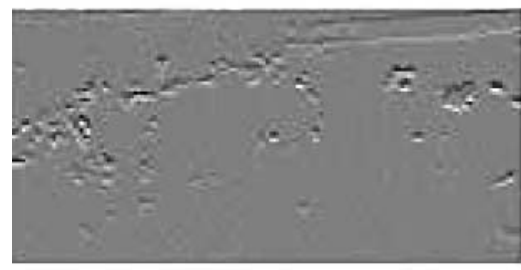

D

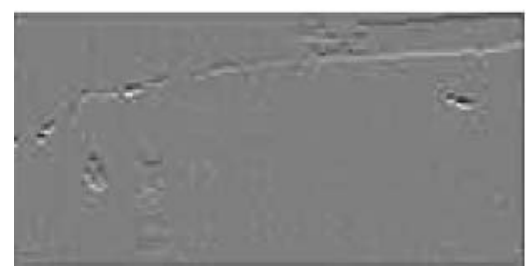

Figure 7 Axial strain maps for aorta images in fig 5 calculated with the following kernels: (A) $21 \times 21$, (B) $31 \times 31$, (C) $41 \times 41$, and (D) $61 \times 61$ pixels.

Strain

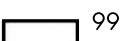

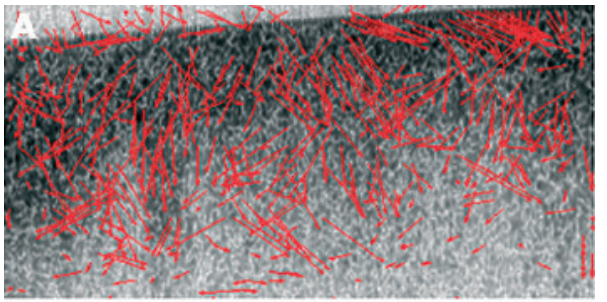
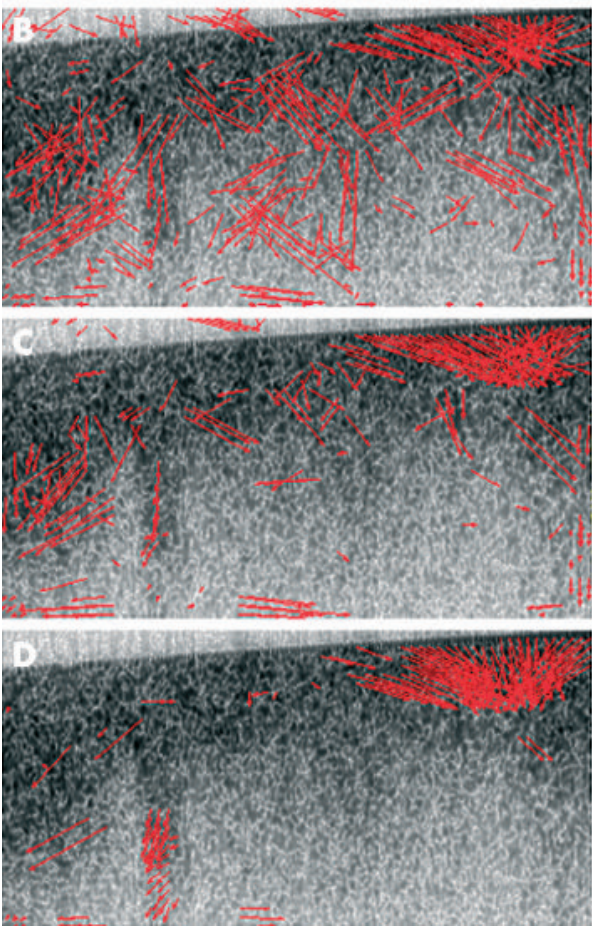

Figure 8 Displacement vectors for aorta images in fig 5 calculated by cross correlation with the following kernels: (A) $21 \times 21$, (B) $31 \times 31$, (C) $41 \times 41$, and (D) $61 \times 61$ pixels.

interest are selected first (for example, charcoal particles or latex spheres on phantom images or plaque areas on arterial images) and the cross correlation is calculated for only selected features. Features can be preselected either manually or by automated image processing segmentation techniques.

In summary, we showed that preselected analysis criteria are critical for the correct interpretation of OCT elastography results. Future work is needed to understand how OCT can be best applied to assessing risk associated with vulnerable plaque.

\section{ACKNOWLEDGEMENTS}

This research is supported in part by the United States National Institutes of Health (contracts NIH-ROl-AR44812, NIH R01 AR46996, NIH R01-HL63953, NIH-1-R01-HL55686, and NIH R01 EB000419) and the Whitaker Foundation (Contract No. 96-0205).

\section{Authors' affiliations}

J Rogowska, N A Patel, M E Brezinski, Orthopedics Department, Brigham and Women's Hospital/Harvard Medical School, 75 Francis Street, Boston, Massachusetts 02115, USA

J G Fujimoto, Department of Electrical Engineering and Computer Science, Massachusetts Institute of Technology, Cambridge, Massachusetts 02139, USA

\section{REFERENCES}

1 Brezinski ME, Tearney GJ, Bouma BE, et al. Optical coherence tomography for optical biopsy: properties and demonstration of vascular pathology. Circulation 1996;93:1206-13. 
2 Brezinski ME, Tearney GJ, Bouma BE, et al. Imaging of coronary artery microstructure with optical coherence tomography. Am J Cardiol 1996;77:92-3.

3 Brezinski ME, Tearney GJ, Weissman NJ, et al. Assessing atherosclerotic plaque morphology: comparison of optical coherence tomography and high frequency ultrasound. Heart 1997;77:397-403.

4 Tearney GJ, Brezinski ME, Boppart SA, et al. Catheter based optical imaging of a human coronary artery. Circulation 1996;94:3013.

5 Patwari P, Weissman NJ, Boppart SA, et al. Assessment of coronary plaque with optical coherence tomography and high frequency ultrasound. Am J Cardiol 2000;85:641-4

6 Fujimoto JG, Boppart SA, Tearney GJ. High resolution in vivo intra-arterial imaging with optical coherence tomography. Heart 1999;82:128-33.

7 Bouma BE, Tearney GJ, Yabushita $\mathrm{H}$, et al. Evaluation of intracoronary stenting by intravascular optical coherence tomography. Heart 2003;89:317-20

8 Jang IK, Bouma BE, Kang DH, et al. Visualization of coronary atherosclerotic plaques in patients using optical coherence tomography: comparison with intravascular ultrasound. J Am Coll Cardiol 2002;39:604-9.

9 Erkamp RQ, Wiggins $P$, Skovoroda AR, et al. Measuring the elastic modulus of small tissue samples. Ultrason Imaging 1998;20:17-28.

10 De Korte CL, Cespedes I, van der Steen AFW, et al. Intravascular elasticity imaging using ultrasound. Ultrasound Med Biol 1997;23:725-46.

11 De Korte CL, van der Steen AFW, Cespedes I, et al. Intravascular ultrasound elastography in human arteries: initial experience in vitro. Ultrasound Med Biol 1998;24:401-9.
12 Ophir J, Cespedes I, Ponnekanti H, et al. Elastography: a quantitative method for imaging the elasticity of biological tissues. Ultrason Imaging $1991 ; 13: 111-34$.

13 Shapo $B M$, Crowe JR, Erkamp RQ, et al. Strain imaging of coronary arteries with intraluminal ultrasound: experiments on an inhomogeneous phantom. Ultrason Imaging 1996;18:173-91.

14 Heers G, Jenkyn T, Dresner MA, et al. Measurement of muscle activity with magnetic resonance elastography. Clin Biomech (Bristol, Avon). 2003; 18:537-42.

15 Manduca A, Oliphant TE, Dresner MA, et al. Magnetic resonance elastography: non-invasive mapping of tissue elasticity. Med Image Anal 2001;5:237-54.

16 Bishop J, Samani A, Sciarretta J, et al. Two-dimensional MR elastography with linear inversion reconstruction: methodology and noise analysis. Phys Med Biol 2000:45:2081-91.

17 Kruse SA, Smith JA, Lawrence AJ, et al. Tissue characterization using magnetic resonance elastography: preliminary results. Phys Med Biol 2000;45:1579-90

18 Muthupillai R, Ehman RL. Magnetic resonance elastography. Nat Med 1996;2:601-3.

19 Schmitt JM. OCT elastography: imaging microscopic deformation and strain in tissue. Opt Express 1998;3:199-211.

20 Rogowska J, Patel N, Fujimoto JG, et al. OCT elastography of vascular tissue: importance of cross-correlation kernel size. Proceedings of the OSA Biomedical Topical Meetings, Advances in Optical Imaging and Photon Migration, Miami, 2002:PD20-1-PD20-3.

\section{IMAGES IN CARDIOLOGY}

\section{Embolism of thrombus in the right coronary artery to the left anterior descending artery in a woman with a single coronary artery}

\begin{abstract}
64 year old woman with chest pain and ECG consistent with an inferior myocardial infarction received thrombolysis. There was no evidence of reperfusion and she underwent rescue angioplasty. She was found to have a single coronary artery arising from the right sinus of Valsalva. The right coronary artery (RCA) was occluded by thrombus (panel A). During the first contrast injection a portion of the thrombus was dislodged and travelled across the left main stem (panel B) into the left anterior descending artery (LAD) causing a distal occlusion (panel C). The proximal occlusion of the RCA was successfully treated by thrombectomy (X-sizer, Plymouth, Minnesota, USA) and stenting (Sonic, Velocity $4.0 \times 28 \mathrm{~mm}$ ) with an excellent final angiographic result (panel D), although unfortunately there remained sluggish blood flow in the LAD. The patient required an intra-aortic balloon pump and inotropic support for 24 hours. The peak creatine kinase concentration was $5500 \mathrm{u} / \mathrm{l}$.

Single coronary arteries are a recognised but rare anomaly with an incidence of around $0.02 \%$. They may be clinically significant when a major branch passes between the aorta and the right ventricular outflow tract as was found in this case. This particular anomaly is associated with sudden cardiac death possibly due to compression between the major vessels. In our middle aged patient it is likely that the thrombus in the proximal RCA was a consequence of underlying atherosclerosis. Unfortunately occlusion of the distal LAD resulted from embolised thrombus dislodged from the RCA by contrast injection-an extremely rare event.
\end{abstract}

S J Leslie

I R Starkey

s.j.leslie@ed.ac.uk

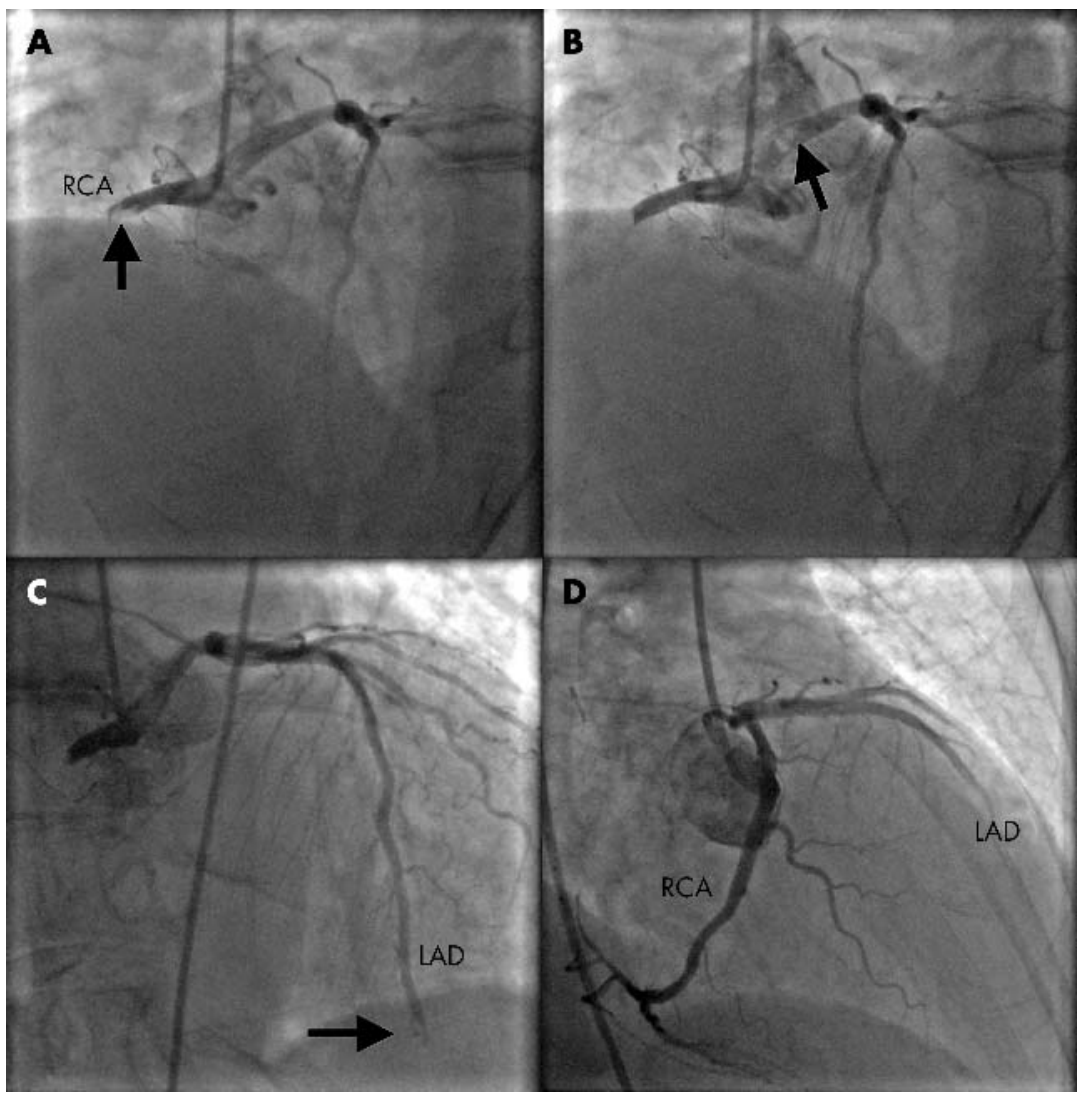

